\title{
Towards Fully Automated Systems for the Generation of Very High Order Modes in Oversized Waveguides
}

\author{
$\underline{\text { T. Ruess }}^{1}$, K. A. Avramidis ${ }^{1}$, M. Fuchs, G. Gantenbein ${ }^{1}$, S. Illy ${ }^{1}$, F.-C. Lutz ${ }^{1}$, A. Marek ${ }^{1}$, S. Ruess ${ }^{1,2}$, \\ T. Rzesnicki ${ }^{1}$, M. Thumm ${ }^{1,2}$, D. Wagner ${ }^{3}$, J. Weggen ${ }^{1}$, and J. Jelonnek ${ }^{1,2}$ \\ ${ }^{1}$ IHM, ${ }^{2}$ IHE, Karlsruhe Institute of Technology (KIT), Kaiserstr. 12, D-76131 Karlsruhe, Germany \\ ${ }^{3}$ Max Planck Institute for Plasma Physics, Boltzmannstr. 2, D-85748 Garching, Germany
}

\section{Introduction}

Fusion plasma experiments require very powerful heating systems to achieve the needed high plasma temperatures. For Electron Cyclotron Resonance Heating and Current Drive (ECRH\&CD), the gyrotron oscillator is the only known source which is capable to produce a megawatt-level RF output power [1] in the range from $30 \mathrm{GHz}$ up to $300 \mathrm{GHz}$ for a possible future DEMO EC heating system. The excitation of the main operating mode in the gyrotron cavity and the separation of this mode from the different possible competing modes is a very challenging task due to the very high order of that modes. A similar problem exists for the excitation of the wanted high-order mode in low-power test systems to validate the quasi-optical output system of that kind of gyrotrons. But, the availability of such a test system is vital during the gyrotron design and validation phases. Particularly, the launcher of the quasi-optical output coupler, which converts the rotating high-order operating cavity mode into a linearly polarized Gaussian output beam, is the critical component here. The existing test set-up at Karlsruhe Institute of Technology (KIT) which basic idea bases on [2], includes a quasi-optical mode generator using fully manually operated mechanical sliding units [3]. Obviously, those manual sliding units suffer from a large hysteresis, hence, require a very time-consuming adjustment. Here the first steps towards a fully automated test system are presented.

\section{First steps towards a fully automated mode generator setup}

Moving towards a fully automated test system at KIT required three major steps: the introduction of a fully automated mechanical adjustment of the mode generator, the replacement of the frequency multiplier and harmonic mixer [4] by a new vector network analyzer which is even capable to cover the complete frequency range from $140 \mathrm{GHz}$ to $330 \mathrm{GHz}$ using different frequency extension modules and an intelligent algorithm which finds autonomous a setup configuration to excite the correct mode.

The sliding units were to replace by two precision linear stages, as depicted in Fig. 1, which can be handled by a computer. It allows to run experiments including an adjustment of the quasi-parabolic (q.-p.) mirror system without any human attendance.

The proposed system has been validated by the excitation of the $\mathrm{TE}_{28,8}$-mode at $140 \mathrm{GHz}$ used for the
$1 \mathrm{MW}, \mathrm{CW}$ gyrotrons for the stellerator W7-X at IPP Greifswald, Germany [5]. Recently, an upgraded 1.5 MW version of this gyrotron, with the possibility of operation also at $175 \mathrm{GHz}$, is under discussion. Therefore, a mode generator cavity for the $\mathrm{TE}_{28,10} /$

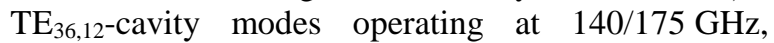
respectively, has been designed, fabricated and successfully tested [6]. The cavity of the generator contains an inner conductor and is especially designed for the low power measurement setup. Its design has been studied and optimized employing a scattering matrix code [7]. A frequency shift occurs due to the perforated midsection area. First results are shown in [6], where the operation frequency are determined at $140.155 \mathrm{GHz}$ and $175.992 \mathrm{GHz}$. The remaining frequency shift of around $155 \mathrm{MHz}$ can be eliminated by increasing the radius of the inner rod which leads to a lower eigenvalue and thus a lower frequency. The measured $\mathrm{TE}_{28,10}$ mode pattern at $140.01 \mathrm{GHz}$ is shown in Fig. 2 and 3. The highest mode content is determined by $92.2 \%$ and the counter-rotating amount is calculated to be $0.57 \%$. The designed quality factor is 2506 and is in very good agreement with the measured 2522. The adjustment of this mode was done using the old setup, as well. The comparison show, that a time saving of more than $94 \%$ is achieved using the new setup with the advanced algorithm.

Recently, programs for the determination of the frequency, acquisition of the mode pattern and the mode pattern evaluation are running separately. In the future, those programs will run sequential and an already written intelligent algorithm makes the decision if the acquired mode pattern is matching to the theoretical one or not. The algorithm has two main functions, namely (i) finding the ideal position for the q.-p. mirror and (ii) the determination of the mode and its quality. Regarding point (ii), five techniques are

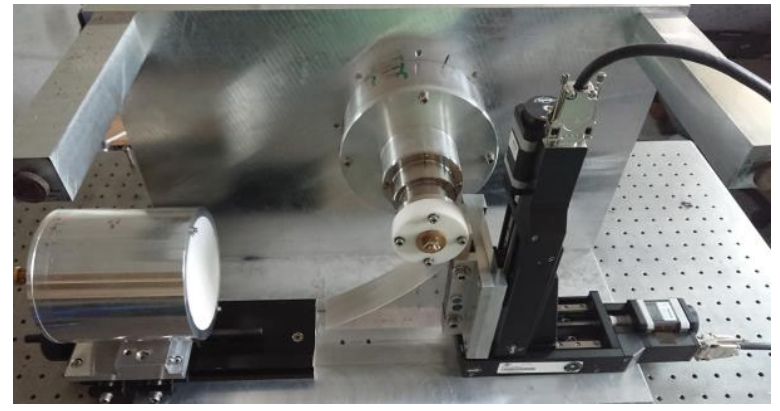

Fig. 1. Depiction of the mode generator setup. 
Amplitude $[\mathrm{dB}]$

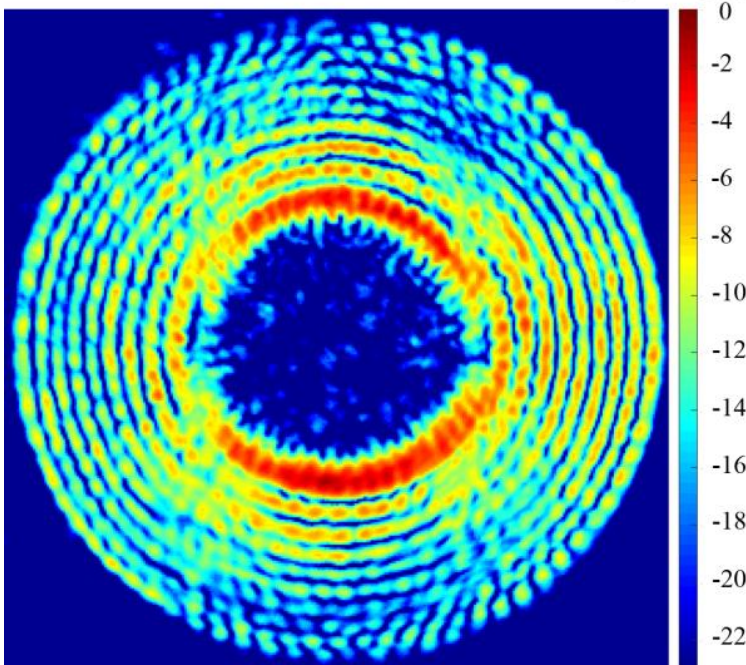

Fig. 2. Amplitude pattern of the $\mathrm{TE}_{28,10-\text { mode at }}$ $140.01 \mathrm{GHz}$ with a resolution of the measurement arm of $0.2 \times 0.2 \mathrm{~mm}$

implemented. It can determine the radial index by counting the number of rings and the azimuthal mode index by evaluating the phase jumps. Further, the scalar mode content, the quality factor and the amount of the counter-rotating mode are calculated.

\section{Tolerance study of the quasi-parabolic mirror}

The adjustment of the q.-p. mirror is the most important parameter for a correct excitation of the mode. A tolerance study has been started proving the required adjustment accuracy. The precision linear tables under use have a position error of $<25 \mu \mathrm{m} / 100 \mathrm{~mm}$ and a repetition failure (bidirectional) of $<15 \mu \mathrm{m}$. For this study, different q.p. mirror positions are tested and the scalar mode content and the amount of the counter-rotating mode is calculated at each position and summarized in Tab. 1. Any deviation in $\mathrm{x}$ - and $\mathrm{y}$-direction can be assumed and measured using the two precision linear tables. The values $\Delta \mathrm{x}$ and $\Delta \mathrm{y}$ are the differences between the actual and the ideal position. A deviation

Tab. 1. Summary of the tolerance study regarding different positions of the q.-p. mirror in respect to the ideal one.

\begin{tabular}{|c|c|c|c|c|}
\hline ID & $\begin{array}{c}\Delta \mathrm{x} \\
{[\mathrm{mm}]}\end{array}$ & $\begin{array}{c}\Delta \mathrm{y} \\
{[\mathrm{mm}]}\end{array}$ & $\begin{array}{c}\text { Scalar mode } \\
\text { content [\%] }\end{array}$ & $\begin{array}{c}\text { counter- } \\
\text { rotating } \\
\text { mode [\%] }\end{array}$ \\
\hline 1 & 0.0 & 0.0 & 92.2 & 0.57 \\
\hline 2 & -0.8 & 0.0 & 88.6 & 4.15 \\
\hline 3 & -0.8 & +0.7 & 90.2 & 1.43 \\
\hline 4 & -0.4 & 0.0 & 91.0 & 1.25 \\
\hline 5 & -0.4 & +0.7 & 92.0 & 1.13 \\
\hline 6 & 0 & +0.7 & 92.0 & 1.05 \\
\hline 7 & -0.2 & -0.2 & 91.5 & 1.51 \\
\hline 8 & -0.2 & 0.0 & 91.1 & 1.35 \\
\hline 9 & -0.8 & -0.2 & 92.1 & 1.00 \\
\hline 10 & -0.8 & 0.0 & 92.1 & 1.05 \\
\hline
\end{tabular}

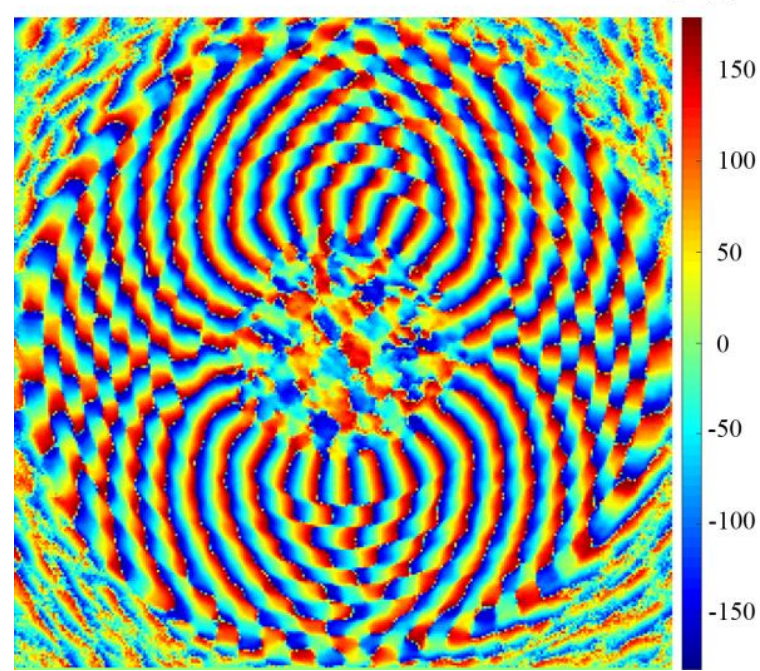

Fig. 3. Phase diagram of the $\mathrm{TE}_{28,10}$ mode pattern at $140.01 \mathrm{GHz}$ with a resolution of the measurement arm of $0.2 \times 0.2 \mathrm{~mm}$

of $\Delta \mathrm{x}=3 \mathrm{~mm}$ leads to a mode pattern where several modes are superimposed and no dominant mode could be identified. A deviation of $\Delta x=1.5 \mathrm{~mm}$ leads to a mode pattern where several modes are superimposed as well, but the $\mathrm{TE}_{28,10}$-mode could be identified as dominant. Nevertheless, the quality is not sufficient. In general, a deviation of the ideal mirror position reduces the scalar mode content and increases the amount of the counter-rotating mode. But, the impact of a deviation of roughly $0.8 \mathrm{~mm}$ is not incredible high for this specific cavity, as can be seen in Tab. 1 . In the next steps, further tolerance analysis will be made.

\section{References}

1. Rzesnicki, T. et al., 2.2-MW record power of the 170$\mathrm{GHz}$ european pre-prototype coaxial-cavity gyrotron for ITER // IEEE Trans, on Plasma Science, Vol. 38, No. 6, pp. 1141-1149, 2010.

2. Alexandrov, N. L. et al., Low-power excitation of gyrotron-type modes in a cylindrical waveguide using quasi-optical techniques // Int. Journal of Electronics, 79, pp. 215-216, 1995.

3. Ruess, T. et al, 2018 status on the measurement capabilities for fusion gyrotrons at KIT/IHM // $30^{\text {th }}$ Joint Russian-German Meeting on ECRH and Gyrotrons, EPJ Web of Conferences (2018).

4. Losert, M., Jin, J. and Rzesnicki, T. RF beam parameter measurements of quasi-optical mode converters in the mw range // IEEE Trans. On Plasma Science, vol. 41, no. $3,2013$.

5. Braune, $H$. et al., Gyrotron operation during the first W7-X campaign - handling and reliability // in Proc. IRMMW, Copenhagen, Denmark, pp. 1-2, 2013.

6. Avramidis, $K$. A. et al., Studies towards an upgraded $1.5 \mathrm{MW}$ gyrotron for W7-X // $20^{\text {th }}$ Joint Workshop on Electron Cyclotron Emission (ECE) and Electron Cyclotron Resonance Heating (ECRH), Greifswald, Germany, May 2018.

7. Wagner, D., Pretterebner, J., Thumm, M., Design of coaxial gyrotron cavities using a scattering matrix code $/ / 5^{\text {th }}$ Joint Russian-German Meeting on ECRH and Gyrotrons, pp. 555-565, 1993. 